\title{
Quantum Interference Control of Carriers and Currents in Zincblende Semiconductors based on Nonlinear Absorption Processes
}

\author{
Rodrigo A. Muniz, ${ }^{1, *}$ Cuauhtémoc Salazar,,${ }^{1} \dagger$ Kai Wang, ${ }^{2,}{ }^{\ddagger}$ S. T. Cundiff, ${ }^{2}$ and J. E. Sipe ${ }^{1, \S}$ \\ ${ }^{1}$ Department of Physics, University of Toronto, Toronto, Ontario M5S 1A\%, Canada \\ ${ }^{2}$ Department of Physics, University of Michigan, Ann Arbor, Michigan 48109, USA
}

(Dated: June 20, 2019)

\begin{abstract}
Quantum interference between optical absorption processes can excite carriers with a polarized distribution in the Brillouin zone depending on properties of the incident optical fields. The polarized distribution of carriers introduces a current that can be controlled by the phases and polarizations of the incident optical fields. Here we study the quantum interference of 2- and 3-photon absorption processes in AlGaAs. We present theoretical predictions for carrier and current injection rates considering different frequencies, phases, and polarizations of the incident fields. We also discuss the important features that result from only nonlinear optical processes being involved, which leads for instance to a sharper distribution of carriers in the Brillouin zone.
\end{abstract}

\section{INTRODUCTION}

Quantum interference between different optical processes arises when two optical beams of different frequencies can lead to the same transition. It has been used to study photoionization of molecular systems [1-6], and asymmetric photoejection in semiconductors [7-11]. In a crystal, amplitudes for different optical processes leading to electron-hole excitations can interfere constructively in some regions of the Brillouin zone (BZ), and destructively in others. By controlling the polarizations and phases of the incident fields, it is possible to excite carriers in selected localized regions of the BZ. Such Quantum Interference Control (QuIC), using 1- and 2-photon absorption processes (1+2 QuIC), has been used for current injection in semiconductors [12-14], graphene [1517], topological insulators [18-20], and transition metal dichalcogenides [21-23], as well as spin current injection in semiconductors [24-28]. It has also been theoretically investigated for current injection in graphene nanoribbons [29], spin currents in topological insulators [18], and spin and valley currents [30] in transition metal dichalcogenides [21, 23].

In crystalline materials, every instance of QuIC studied to date has involved 1- and 2-photon absorption processes, partly because phase related optical fields of frequencies $\omega$ and $2 \omega$ can be conveniently achieved by second harmonic generation, while fractional ratios of the frequencies are harder to obtain. Currents injected via $1+2$ QuIC have been exploited to determine parameters of the optical fields responsible for their injection [31-33]. This method has found application in the measurement

\footnotetext{
* Current address: Department of Electrical Engineering and Computer Science, University of Michigan, Ann Arbor, MI 48109, USA

$\dagger$ Current address: Department of Physics, University of Warwick, Coventry, CV4 7AL, United Kingdom

$\ddagger$ Current address: Department of Physics, Texas A\&M University, College Station, TX 77843, USA

$\S$ email: sipe@physics.utoronto.ca
}

$[34,35]$ and stabilization of the carrier-envelope phase of a train of octave-spanning laser pulses [36-38]. However, phase-coherent frequency combs can also be used to study more general $M+N$ QuIC, especially for fractional ratios $N / M<2$ that only require a narrower frequency range of the comb. Thus the use of optical frequency combs for QuIC experiments presents an opportunity to separately study several nonlinear optical processes in semiconductors, which cannot easily be done using simple harmonic generation, as it only produces frequencies that are integer multiples.

In this article we present a theoretical study of QuIC with 2- and 3-photon processes in AlGaAs. The injection rates of $1+2 \mathrm{QuIC}$ correspond to divergences in the thirdorder electric susceptibility, $\chi^{(3)}$, while the injection rates of $2+3$ QuIC correspond to divergences in the fifth-order electric susceptibility, $\chi^{(5)}$. Although sophisticated calculations of $\chi^{(3)}$ have been performed for similar analyses in 1+2 QuIC [39-41], here we use a Fermi Golden Rule approach in the perturbative regime, as it allows us to focus only on the resonant processes we are interested, and ignore many other non-resonant processes described by $\chi^{(5)}$. Such an approach has served as a basis for further studies of $1+2$ QuIC $[12,16]$, and in this article we follow it to provide a first step in elucidating $2+3 \mathrm{QuIC}$ as well. We derive expressions for the optical injection coefficients at the initial time when the fields are incident, and evaluate them for different stoichiometries of AlGaAs using a 30 -band $\boldsymbol{k} \cdot \boldsymbol{p}$ model. We compute all the symmetry-allowed injection coefficients corresponding to different polarizations of the incident fields, and analyze their frequency dependence over a range where the injection of carriers that do not contribute to the current is suppressed. That is, considering 3-photon absorption of photons at energy $\hbar \omega$ and 2-photon absorption of photons at energy $3 \hbar \omega / 2$, we require $2 \hbar \omega$ to be less than the band gap. The alloy AlGaAs is an ideal material for $2+3$ QuIC, as its stoichiometry can be chosen to yield a band gap appropriate for the available laser wavelengths. Experiments demonstrating $2+3 \mathrm{QuIC}$ of photocurrents in AlGaAs are being reported in another article [42]. 
As would be expected, there are qualitative differences between $2+3 \mathrm{QuIC}$ and $1+2 \mathrm{QuIC}$. For instance, there is a change of sign in the current injection coefficient for different frequencies, which is due to an interplay between intraband and interband processes contributing to 3-photon absorption. We also find that 2+3 QuIC leads to sharper distributions of carriers in the BZ than $1+2$ QuIC. The sharper distribution of carriers leads to a higher swarm velocity, which is a desirable feature for photocurrents, and it also opens the possibility of exciting carriers in semiconductors in a tailored fashion.

The outline of this article is the following: In Sec. II we present a method to compute the optical injection rates for a generic material. In Sec. III we describe the model used for AlGaAs. In Sec. IV we present our results for carrier and current injection from 2- and 3-photon absorption (2PA and $3 \mathrm{PA}$ ) processes in AlGaAs. We also discuss the efficiency of the current injection by analyzing the swarm velocity, and computing the optimal laser intensities. In Sec. V we discuss the implications of our results and present our conclusions. We list the independent components of the optical injection tensors for zincblende lattice symmetry in the Appendix.

\section{OPTICAL INJECTION RATES}

In this article, we restrict ourselves to computing the optical injection rates at the initial time when the fields are incident. We neglect scattering processes, carrier acceleration in the BZ, and several other effects that later influence the dynamics of the injected carriers. We thus use a Fermi Golden Rule approach [14, 18] as it is adequate for computing transition rates. Other approaches based on solving equations of motion [39-41] for the system have been used for $1+2 \mathrm{QuIC}$, and they can be straightforwardly extended to $2+3$ QuIC.

Assuming the independent particle approximation, we consider a system described by a Hamiltonian $\mathcal{H}_{0}$ in the absence of any external perturbation, so the full Hamiltonian $\mathcal{H}(t)$ in the presence of the external perturbation $\mathcal{V}_{\text {ext }}(t)$ is $\mathcal{H}(t)=\mathcal{H}_{0}+\mathcal{V}_{\text {ext }}(t)$, where in the basis of eigenstates of $\mathcal{H}_{0}$,

$$
\begin{aligned}
\mathcal{H}_{0} & =\sum_{n \boldsymbol{k}} \hbar \omega_{n \boldsymbol{k}} a_{n \boldsymbol{k}}^{\dagger} a_{n \boldsymbol{k}}, \\
\mathcal{V}_{\text {ext }}(t) & =\sum_{m n \boldsymbol{k}} a_{m \boldsymbol{k}}^{\dagger} V_{m n \boldsymbol{k}}(t) a_{n \boldsymbol{k}},
\end{aligned}
$$

where $|n \boldsymbol{k}\rangle=a_{n \boldsymbol{k}}^{\dagger} \mid$ vac $\rangle$ indicates a Bloch state corresponding to band $n$, with crystal momentum $\boldsymbol{k}$, and energy $\hbar \omega_{n \boldsymbol{k}}$. In the interaction picture, the creation and annihilation fermion operators are $a_{n \boldsymbol{k}}^{\dagger}(t)=a_{n \boldsymbol{k}}^{\dagger} e^{i \omega_{n} t}$ and $a_{n \boldsymbol{k}}(t)=a_{n \boldsymbol{k}} e^{-i \omega_{n} t}$, the external perturbation operator is $\mathcal{V}_{\mathrm{I}}(t)=e^{i \mathcal{H}_{0} t / \hbar} \mathcal{V}_{\text {ext }}(t) e^{-i \mathcal{H}_{0} t / \hbar}$, and the timeevolution operator can be expanded as

$$
\mathcal{U}(t)=1+\sum_{N=1}^{\infty} \int_{-\infty}^{t} \frac{d t_{N}}{i \hbar} \mathcal{V}_{\mathrm{I}}\left(t_{N}\right) \cdots \int_{-\infty}^{t_{2}} \frac{d t_{1}}{i \hbar} \mathcal{V}_{\mathrm{I}}\left(t_{1}\right)
$$

and the terms of each order in $\mathcal{V}_{\text {ext }}$ can be obtained from the previous one by

$$
\begin{aligned}
\mathcal{U}_{N}(t)= & \int_{-\infty}^{t} \frac{d t_{N}}{i \hbar} \sum_{m n \boldsymbol{k}} \\
& a_{m \boldsymbol{k}}^{\dagger}\left(t_{N}\right) V_{m n \boldsymbol{k}}\left(t_{N}\right) a_{n \boldsymbol{k}}\left(t_{N}\right) \mathcal{U}_{N-1}\left(t_{N}\right),
\end{aligned}
$$

where $\omega_{m n \boldsymbol{k}}=\omega_{m \boldsymbol{k}}-\omega_{n \boldsymbol{k}}$, and $\mathcal{U}_{0}(t)=1$. We are interested in the excitation of an electron from a valence band $v$ to a conduction band $c$ due to the external field. This excited state is $|c v \boldsymbol{k}\rangle=a_{c \boldsymbol{k}}^{\dagger} a_{v \boldsymbol{k}}|\mathrm{gs}\rangle$, where $|\mathrm{gs}\rangle$ is the eigenstate of $\mathcal{H}_{0}$ with filled valence bands. The state of the system is described by

$$
\begin{aligned}
|\psi\rangle & =\mathcal{U}(t)|g \mathrm{~g}\rangle \\
& =\gamma_{0}|\mathrm{gs}\rangle+\sum_{c v \boldsymbol{k}} \gamma_{c v \boldsymbol{k}}(t)|c v \boldsymbol{k}\rangle+\ldots
\end{aligned}
$$

where the coefficient

$$
\gamma_{c v \boldsymbol{k}}(t)=\langle c v \boldsymbol{k}|\mathcal{U}(t)| \mathrm{gs}\rangle
$$

indicates the degree to which the system has been excited into the $|c v \boldsymbol{k}\rangle$ state, so they allow us to compute injection rates. We point out that the $\gamma_{c v \boldsymbol{k}}(t)$ coefficients are related to a single-particle density matrix $\rho_{m n}(t)=\left\langle\psi\left|a_{m \boldsymbol{k}}^{\dagger}(t) a_{n \boldsymbol{k}}(t)\right| \psi\right\rangle$, which could be used for computing $\chi^{(5)}$, but that is a much more complicated calculation and it includes several non-resonant effects that are not the focus of our study. Thus we use a simpler parametrization for the states of the system in terms of $\gamma_{c v \boldsymbol{k}}(t)$.

For a full Hamiltonian $\mathcal{H}(t)$ that follows from a Hamiltonian for a single particle of the form

$$
\begin{aligned}
\mathscr{H}(\boldsymbol{x}, \boldsymbol{p} ; t)= & \frac{1}{2 m}(\boldsymbol{p}-e \boldsymbol{A}(t))^{2} \\
& +\mathscr{H}_{S O}(\boldsymbol{x}, \boldsymbol{p}-e \boldsymbol{A}(t))+\mathscr{V}_{\text {lat }}(\boldsymbol{x}),
\end{aligned}
$$

where $\boldsymbol{x}$ and $\boldsymbol{p}$ are position and momentum operators, $H_{S O}$ is the spin-orbit term, and $\mathscr{V}_{\text {lat }}(\boldsymbol{x})$ is the lattice potential energy. Here we neglect a contribution to the interaction that is solely a function of time $\left(\sim[A(t)]^{2}\right)$, for it will not lead to any transitions, and we work in a gauge where the electric field $\boldsymbol{E}(t)$, assumed independent of position, is fully described by the vector potential $\boldsymbol{A}(t)$. We point out that for a general $\boldsymbol{A}$ dependent on the position, neglecting the $[A]^{2}$ term in the Hamiltonian would be problematic. The interaction term in the Hamiltonian takes the form $\mathscr{V}_{\text {ext }}(t)=-e \mathfrak{v} \cdot \boldsymbol{A}(t)$, where $e=-|e|$ is the charge of the electron and $\mathfrak{v}=-e^{-1} \partial \mathscr{H} / \partial \boldsymbol{A}$ is the velocity operator. Indeed, the interaction is of the form we consider for any unperturbed Hamiltonian for a single particle that is at most quadratic in the momentum.

We take the vector potential to be

$$
\boldsymbol{A}(t)=\sum_{\alpha} \boldsymbol{A}_{\alpha} e^{-i\left(\omega_{\alpha}+i \epsilon\right) t}=-\sum_{\alpha} \frac{i}{\omega_{\alpha}} \boldsymbol{E}_{\alpha} e^{-i\left(\omega_{\alpha}+i \epsilon\right) t},
$$


with $\omega_{\alpha}= \pm \omega, \pm 3 \omega / 2$; here $\epsilon \rightarrow 0^{+}$describes turning on the field from $t=-\infty$. The $\gamma_{c v \boldsymbol{k}}(t)$ coefficients can be expanded as $\gamma_{c v \boldsymbol{k}}^{(N)}(t)=\left\langle c v \boldsymbol{k}\left|\mathcal{U}_{N}(t)\right| \mathrm{gs}\right\rangle$ following the expansion (4) of $\mathcal{U}(t)$ for an incident optical field, so we can write the coefficients $\gamma_{c v \boldsymbol{k}}^{(N)}(t)$ as

$$
\gamma_{c v \boldsymbol{k}}^{(N)}(t)=\mathscr{R}_{c v \boldsymbol{k}}^{(N)} \frac{e^{-i\left(\Omega_{N}-\omega_{c v \boldsymbol{k}}+i \epsilon\right) t}}{\Omega_{N}-\omega_{c v \boldsymbol{k}}+i \epsilon},
$$

where $\Omega_{N}=\omega_{1}+\ldots+\omega_{N}$. The coefficients $\mathscr{R}_{c v \boldsymbol{k}}^{(N)}$ involve the electric field amplitudes $\boldsymbol{E}_{\alpha}$ according to where repeated indices are summed; here superscripts refer to Cartesian indices and subscripts to incident frequency components. For the lower orders we have

$$
\begin{aligned}
R_{c v \boldsymbol{k}}^{(1) a}\left(\omega_{\alpha}\right)= & \sum_{\alpha} \frac{i e}{\hbar \omega_{\alpha}} v_{c v \boldsymbol{k}}^{a}, \\
R_{c v \boldsymbol{k}}^{(2) a b}\left(\omega_{\alpha}, \omega_{\beta}\right)= & \sum_{\alpha \beta} \frac{-e^{2}}{\hbar^{2} \omega_{\alpha} \omega_{\beta}} \\
& \times\left(\sum_{c^{\prime}} \frac{v_{c c^{\prime} \boldsymbol{k}}^{a} v_{c^{\prime} v \boldsymbol{k}}^{b}}{\omega_{\beta}-\omega_{c^{\prime} v \boldsymbol{k}}}-\sum_{v^{\prime}} \frac{v_{c v^{\prime} \boldsymbol{k}}^{b} v_{v^{\prime} v \boldsymbol{k}}^{a}}{\omega_{\beta}-\omega_{c v^{\prime} \boldsymbol{k}}}\right),
\end{aligned}
$$

and

$$
\begin{aligned}
R_{c v \boldsymbol{k}}^{(3) a b d}\left(\omega_{\alpha}, \omega_{\beta}, \omega_{\delta}\right)=\sum_{\alpha \beta \gamma} \frac{i e^{3}}{\hbar^{3} \omega_{\alpha} \omega_{\beta} \omega_{\delta}}\left[\sum_{c^{\prime}} \frac{v_{c c^{\prime} \boldsymbol{k}}^{a}}{\omega_{\alpha}-\omega_{c c^{\prime} \boldsymbol{k}}}\left(\sum_{c^{\prime \prime}} \frac{v_{c^{\prime} c^{\prime \prime} \boldsymbol{k}}^{b} v_{c^{\prime \prime} v \boldsymbol{k}}^{d}}{\omega_{\delta}-\omega_{c^{\prime \prime} v \boldsymbol{k}}}-\sum_{v^{\prime}} \frac{v_{c^{\prime} v^{\prime} \boldsymbol{k}}^{d} v_{v^{\prime} v \boldsymbol{k}}^{b}}{\omega_{\delta}-\omega_{c^{\prime} v^{\prime} \boldsymbol{k}}}\right)\right. \\
-\sum_{v^{\prime}}\left(\sum_{c^{\prime}} \frac{v_{c c^{\prime} \boldsymbol{k}}^{b} v_{c^{\prime} v^{\prime} \boldsymbol{k}}^{d}}{\omega_{\delta}-\omega_{c^{\prime} v^{\prime} \boldsymbol{k}}}-\sum_{v^{\prime \prime}} \frac{v_{c v^{\prime \prime} \boldsymbol{k}}^{d} v_{v^{\prime \prime} v^{\prime} \boldsymbol{k}}^{b}}{\omega_{\delta}-\omega_{c v^{\prime \prime} \boldsymbol{k}}}\right) \frac{v_{v^{\prime} v \boldsymbol{k}}^{a}}{\omega_{\alpha}-\omega_{v^{\prime} v \boldsymbol{k}}} \\
\left.-\sum_{c^{\prime} v^{\prime}}\left(\frac{v_{c v^{\prime} \boldsymbol{k}}^{b} v_{v^{\prime} \boldsymbol{c}^{\prime} \boldsymbol{k}}^{a} v_{c^{\prime} v \boldsymbol{k}}^{d}}{\left(\omega_{\alpha}-\omega_{v^{\prime} c^{\prime} \boldsymbol{k}}\right)\left(\omega_{\delta}-\omega_{c^{\prime} v \boldsymbol{k}}\right)}+\frac{v_{c v^{\prime} \boldsymbol{k}}^{d} v_{v^{\prime} c^{\prime} \boldsymbol{k}}^{a} v_{c^{\prime} v \boldsymbol{k}}^{b}}{\left(\omega_{\delta}-\omega_{c v^{\prime} \boldsymbol{k}}\right)\left(\omega_{\alpha}-\omega_{v^{\prime} c^{\prime} \boldsymbol{k}}\right)}\right)\right] .
\end{aligned}
$$

The expectation value of the density $\langle M\rangle$ of a generic quantity associated with an operator $\mathcal{M}=$ $\sum_{m n \boldsymbol{k}} a_{m \boldsymbol{k}}^{\dagger}(t) M_{m n \boldsymbol{k}} a_{n \boldsymbol{k}}(t)$ can be computed from Eq. (5), and it will have terms independent, linear, and quadratic on $\gamma_{c v \boldsymbol{k}}(t)$. The independent term corresponds to the expectation value $\langle M\rangle$ in the absence of perturbations. The linear terms have accompanying $e^{ \pm i \omega_{c v k} t}$ factors, thus they are fast oscillating and we ignore them as we are interested in computing injection rates. The quadratic term in $\gamma_{c v \boldsymbol{k}}(t)$ then gives us the injection rates, and it is

$$
\begin{aligned}
& \Delta\langle M\rangle= \frac{1}{L^{D}} \sum_{c v c^{\prime} v^{\prime} \boldsymbol{k}}\left\langle c^{\prime} v^{\prime} \boldsymbol{k}|M| c v \boldsymbol{k}\right\rangle \\
& \quad \times \gamma_{c^{\prime} v^{\prime} \boldsymbol{k}}^{*}(t) \gamma_{c v \boldsymbol{k}}(t) e^{i \omega_{c^{\prime} v^{\prime} \boldsymbol{k}} t} e^{-i \omega_{c v \boldsymbol{k}} t} \\
&=\frac{1}{L^{D}} \sum_{c v c^{\prime} v^{\prime} \boldsymbol{k}}\left(M_{c^{\prime} c \boldsymbol{k}} \delta_{v^{\prime} v}-M_{v^{\prime} v \boldsymbol{k}} \delta_{c^{\prime} c}\right) \\
& \quad \times \gamma_{c^{\prime} v^{\prime} \boldsymbol{k}}^{*}(t) \gamma_{c v \boldsymbol{k}}(t) e^{i \omega_{c^{\prime} v^{\prime} \boldsymbol{k}} t} e^{-i \omega_{c v \boldsymbol{k}} t},
\end{aligned}
$$

where $L$ is a normalization length, $D$ is the spatial dimension of the system, and $\Delta\langle M\rangle=\langle M\rangle-\langle M\rangle_{0}$ indicates the change to the expectation value $\langle M\rangle$ due to the perturbation [23]. Since we are interested in the nonoscillatory response of the system, so we focus on the $\Omega_{N}=\Omega_{N^{\prime}}=\Omega$ contributions to Eq. (13). To compute the injection rate $d\langle M\rangle / d t$ associated with Eq. (13), it is important to realize that

$$
\begin{aligned}
& \frac{d}{d t}\left(\gamma_{c^{\prime} v^{\prime} \boldsymbol{k}}^{*}(t) \gamma_{c v \boldsymbol{k}}(t) e^{\left.i \omega_{c^{\prime} v^{\prime} \boldsymbol{k}^{t}} e^{-i \omega_{c v \boldsymbol{k}} t}\right)\left.\right|_{t \rightarrow 0, \epsilon \rightarrow 0}}\right. \\
& =\left.\sum_{N, N^{\prime}} \frac{\mathscr{R}_{c^{\prime} v^{\prime} \boldsymbol{k}}^{\left(N^{\prime}\right) *} \mathscr{R}_{c v \boldsymbol{k}}^{(N)} \times 2 \epsilon}{\left(\Omega-\omega_{c^{\prime} v^{\prime} \boldsymbol{k}}-i \epsilon\right)\left(\Omega-\omega_{c v \boldsymbol{k}}+i \epsilon\right)}\right|_{\epsilon \rightarrow 0} \\
& =\left.\sum_{N, N^{\prime}} \mathscr{R}_{c^{\prime} v^{\prime} \boldsymbol{k}}^{\left(N^{\prime}\right) *} \mathscr{R}_{c v \boldsymbol{k}}^{(N)} 2 \pi \delta\left(\Omega-\omega_{c v \boldsymbol{k}}\right)\right|_{\omega_{c v \boldsymbol{k}}=\omega_{c^{\prime} v^{\prime} \boldsymbol{k}}},
\end{aligned}
$$

where the sums over $N$ and $N^{\prime}$ are restricted to the cases when $\Omega_{N}=\Omega_{N^{\prime}}=\Omega$. The fact that the $\mathscr{R}_{c v \boldsymbol{k}}^{(N)}$ coefficients are always accompanied by $\delta\left(\Omega-\omega_{c v \boldsymbol{k}}\right)$ in the expression for the response allows for substitutions $3 \hbar \omega-\omega_{c v \boldsymbol{k}}=0$ that were used to write $R_{c v \boldsymbol{k}}^{(3) a b d}$ in a simpler way in Eq. (12). The resonance described by Eq. (14) corresponds to a divergence in the electric susceptibility $\chi^{(5)}$, which also describes many non-resonant effects that we are ignoring in this article, as they are much weaker in comparison to resonant ones.

The injection rate of the density of a generic quantity $\langle M\rangle$ can be obtained by taking a time derivative of Eq. (13). Using Eq. (14) and the dependence of

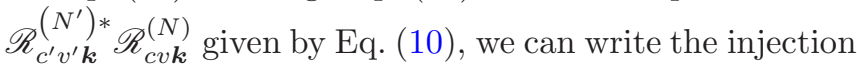
rate of $\langle M\rangle$ due to the interference of an $N^{\prime}$ photon pro- 
cess with an $N$ photon process in terms of a coefficient,

$$
\frac{d}{d t}\langle M\rangle=\mu^{a b d \ldots, p q \ldots}(\Omega) E_{-\alpha}^{a} E_{-\beta}^{b} E_{-\delta}^{d} \ldots E_{\rho}^{p} E_{\sigma}^{q} \ldots+c . c .,
$$

where there are $N^{\prime}$ frequency labels $(\alpha, \beta, \delta, \ldots)$ and $N$ frequency labels $(\rho, \sigma, \ldots)$, and $\Omega_{N}=\Omega_{N^{\prime}}=\Omega$. The injection rate coefficient $\mu^{a b d \ldots, p q \ldots}(\Omega)$ is assembled from the matrix elements $M_{a b \boldsymbol{k}}$ in Eq. (13) and coefficients $R_{c v k}^{(N) p q \cdots}$ in Eq. (14). Taking the continuous momentum limit, we have

$$
\begin{aligned}
\mu^{a b d \ldots, p q \cdots(\Omega)=} & 2 \pi \int \frac{d \boldsymbol{k}}{(2 \pi)^{D}} \sum_{c v c^{\prime} v^{\prime}} \\
& \left(M_{c^{\prime} c \boldsymbol{k}} \delta_{v^{\prime} v}-M_{v^{\prime} v \boldsymbol{k}} \delta_{c^{\prime} c}\right) \delta_{\omega_{c v \boldsymbol{k}}=\omega_{c^{\prime} v^{\prime} \boldsymbol{k}}} \\
& \times R_{c^{\prime} v^{\prime} \boldsymbol{k}}^{\left(N^{\prime}\right) a b d \cdots *} R_{c v \boldsymbol{k}}^{(N) p q \cdots} \delta\left(\Omega-\omega_{c v \boldsymbol{k}}\right) .
\end{aligned}
$$

We will use instances of $\mu^{a b d \ldots, p q \ldots}(\Omega)$ for carrier and current density in this article.

\section{Quantum interference of 2- and 3-photon processes}

The processes of 3PA with frequency $\omega$ and $2 \mathrm{PA}$ with frequency $3 \omega / 2$ can interfere since the total frequency for each of them is $\Omega=3 \omega$, as illustrated in Fig. 1. For such processes the frequencies are all equal in the equations (11) and (12) for the coefficients $R_{c v \boldsymbol{k}}^{(2)}$ and $R_{c v \boldsymbol{k}}^{(3)}$, and symmetrizing their components leads to some simplifications. Using $\omega_{\beta}=\omega_{c v \boldsymbol{k}}-\omega_{\alpha}$ and $\omega_{\alpha}=\omega_{\beta}=3 \omega / 2$ in Eq. (11), the second order coefficient simplifies to

$$
R_{c v \boldsymbol{k}}^{(2) a b}\left(\frac{3 \omega}{2}, \frac{3 \omega}{2}\right)=\frac{-4 e^{2}}{9 \hbar^{2} \omega^{2}} \sum_{m} \frac{v_{c m \boldsymbol{k}}^{a} v_{m v \boldsymbol{k}}^{b}}{\left(\frac{3 \omega}{2}-\omega_{m v \boldsymbol{k}}\right)}
$$

and using $\omega_{\beta}+\omega_{\gamma}=\omega_{c v \boldsymbol{k}}-\omega_{\alpha}$ and $\omega_{\alpha}=\omega_{\beta}=\omega_{\gamma}=\omega$ in Eq. (12), the third order coefficient simplifies to

$$
R_{c v \boldsymbol{k}}^{(3) a b d}(\omega, \omega, \omega)=\frac{i e^{3}}{\hbar^{3} \omega^{3}} \sum_{m n} \frac{v_{c m \boldsymbol{k}}^{a} v_{m n \boldsymbol{k}}^{b} v_{n v \boldsymbol{k}}^{d}}{\left(\omega-\omega_{c m \boldsymbol{k}}\right)\left(\omega-\omega_{n v \boldsymbol{k}}\right)}
$$

Notice that the denominators in Eqs. (17) and (18) are minimal for $m, n=c, v$, so the dominant contributions to $R_{c v \boldsymbol{k}}^{(2)}$ always involve intraband velocity matrix elements, while $R_{c v \boldsymbol{k}}^{(3)}$ also has contributions from interband velocity matrix elements [43]. Intraband velocity matrix elements are associated with the corresponding band dispersion, $v_{n n \boldsymbol{k}}^{a}=\partial_{\boldsymbol{k}}^{a} \omega_{n \boldsymbol{k}}$, which vanishes at the $\boldsymbol{k}$ point corresponding to the bandgap. Thus $R_{c v \boldsymbol{k}}^{(2)}$ is zero for total photon energies corresponding to the band gap, and increases for larger excess photon energies. The dependence of $R_{c v \boldsymbol{k}}^{(3)}$ on the total photon energy is different, as it depends on both interband and intraband velocity matrix elements. For total photon energies just above the gap, $R_{c v \boldsymbol{k}}^{(3)}$ is determined mainly by the interband matrix elements, but as the photon excess energy increases $R_{c v \boldsymbol{k}}^{(3)}$ becomes dominated by the intraband matrix elements, since the electronic transitions occur at $\boldsymbol{k}$ points with larger band dispersion.

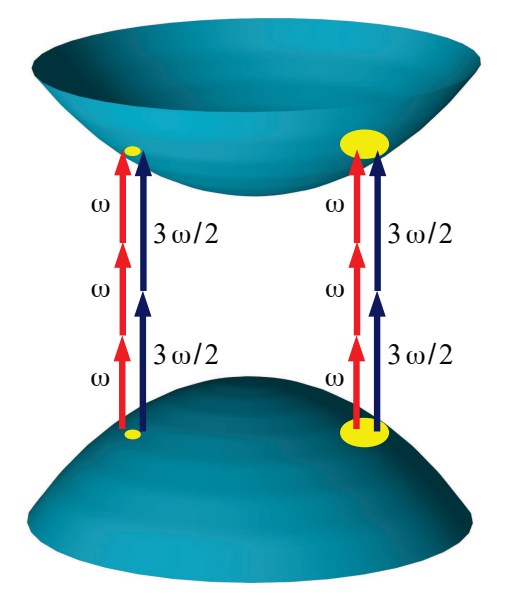

FIG. 1. Depiction of $2+3$ QuIC showing the destructive (left) and constructive (right) interference in different regions of the Brillouin zone.

The injection rate coefficients corresponding to the interference of 2- and 3-photon processes can then be computed from Eqs. (16) as

$$
\begin{aligned}
\mu_{2+3}^{a b d, f g}(\Omega)= & 2 \pi \int \frac{d \boldsymbol{k}}{(2 \pi)^{D}} \sum_{c v c^{\prime} v^{\prime}}\left(M_{c^{\prime} c \boldsymbol{k}} \delta_{v^{\prime} v}-M_{v^{\prime} v \boldsymbol{k}} \delta_{c^{\prime} c}\right) \\
& \times \delta_{\omega_{c v \boldsymbol{k}}=\omega_{c^{\prime} v^{\prime} \boldsymbol{k}}} R_{c^{\prime} v^{\prime} \boldsymbol{k}}^{(3) a b d *} R_{c v \boldsymbol{k}}^{(2) f g} \delta\left(\Omega-\omega_{c v \boldsymbol{k}}\right) .
\end{aligned}
$$

For the plots in the next sections we use a frequency broadening $\Delta$ corresponding to $\hbar \Delta=13 \mathrm{meV}$.

The factor $R_{c^{\prime} v^{\prime} \boldsymbol{k}}^{(3) a b d *} R_{c v \boldsymbol{k}}^{(2) f g}$ changes sign under a transformation $\boldsymbol{k} \rightarrow-\boldsymbol{k}$, resulting in constructive versus destructive interference in opposite points of the Brillouin zone. In Fig. 1 we illustrate constructive versus destructive interference of 2 - and 3 -photon processes at opposite points in the Brillouin zone.

\section{ELECTRONIC MODEL OF AIGaAs}

We use a 30 -band $\boldsymbol{k} \cdot \boldsymbol{p}$ model for computing the electronic bands. The model has free parameters associated with energies and momentum matrix elements at the $\Gamma$ point, and the parameters are adjusted to match the experimental results for band energies from $-5 \mathrm{eV}$ to $4 \mathrm{eV}$, such that computations of optical absorption coefficients are expected to be reliable for photon energies up to $6 \mathrm{eV}$.

Using the $\Gamma$ point as the expansion point for a $\boldsymbol{k} \cdot \boldsymbol{p}$ model, the effective Hamiltonian that acts only on the periodic part of an energy eigenfunction of crystal momentum $\boldsymbol{k}$ is

$$
H_{\mathrm{eff}}=H+\frac{\hbar}{m} \boldsymbol{k} \cdot \boldsymbol{p}+\frac{\hbar^{2} k^{2}}{2 m},
$$



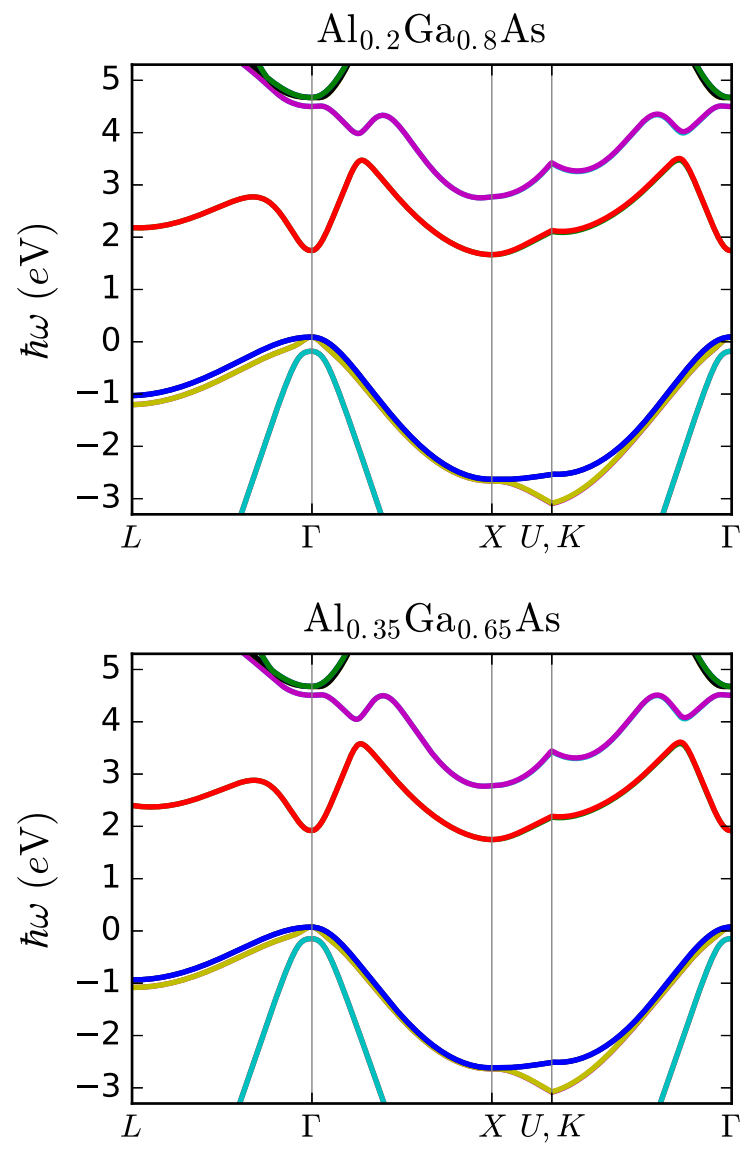

FIG. 2. Electronic bandstructure of AlGaAs for two stoichiometries. For all the stoichiometries $\alpha$ in the range that we consider, $0.18 \lesssim \alpha \lesssim 0.38$, the band structures are vere similar, and their main difference is their bandgap.

where $H$ is the Hamiltonian (7) with the vector potential set equal to zero; in this model [44] we neglect the $k$ dependence of the effective spin-orbit term. The second term on the right-hand-side is the usual $\boldsymbol{k} \cdot \boldsymbol{p}$ contribution, and the last term is the contribution to the kinetic energy only due to the lattice momentum. The basis of states has 8 sets [44], 4 of them corresponding to the $\Gamma_{1}$ representation of the point group $T_{d}$ (or $43 m$ ), 3 corresponding to the $\Gamma_{4}$ representation, and 1 to the $\Gamma_{3}$ representation. The $\Gamma_{1}$ representation has only 1 state, $\Gamma_{4}$ has 3 states, and $\Gamma_{3}$ has 2 states, so in total we have $4 \times 1+3 \times 3+1 \times 2=15$ states before considering spin; we denote these states as $|A\rangle,|B\rangle$, etc. Tensor products of these are taken with spin states to get 30 states in all. Terms $\left\langle A\left|H_{\text {eff }}\right| B\right\rangle$ are then $2 \times 2$ matrices, and take the form

$$
\begin{aligned}
\left\langle A\left|H_{\mathrm{eff}}\right| B\right\rangle= & E_{A} \delta_{A B} \sigma_{0}+\frac{i}{3} \boldsymbol{\Delta}_{A B} \cdot \boldsymbol{\sigma} \\
& +i \boldsymbol{P}_{A B} \cdot \boldsymbol{k} \sigma_{0}+\frac{\hbar^{2} k^{2}}{2 m} \delta_{A B} \sigma_{0},
\end{aligned}
$$

where $\sigma_{0}$ is the unit $2 \times 2$ matrix and the components of $\sigma$ are the usual Pauli matrices. The free parameters of the model are the energies $E_{A}$, the matrix elements of the spin-orbit term $\Delta_{A B}$, and the matrix elements of the momentum operator $\boldsymbol{P}_{A B}$. Since the basis for the states is the same at every $\boldsymbol{k}$ point [45], the corresponding $2 \times 2$ matrices corresponding to the velocity operator $\langle A|\mathfrak{v}| B\rangle$ are diagonal in the spin sector,

$$
\begin{aligned}
\left\langle A\left|\mathfrak{v}^{a}\right| B\right\rangle & =\frac{1}{\hbar} \frac{\partial}{\partial k^{a}}\left\langle A\left|H_{\mathrm{eff}}\right| B\right\rangle \\
& =\left(\frac{i}{\hbar} P_{A B}^{a}+\frac{\hbar k^{a}}{m} \delta_{A B}\right) \sigma_{0},
\end{aligned}
$$

from which the matrix elements of the velocity operator between the energy eigenstates can be determined.

For GaAs [44] and AlAs [46] we use reported parameters adjusted for room temperature, while the parameters for $\mathrm{Al}_{\alpha} \mathrm{Ga}_{1-\alpha} \mathrm{As}$ are obtained from a linear interpolation according to the stoichiometry. This approximation is accurate within an energy tolerance corresponding to room temperature [47]. The chosen parameters lead to effective masses, $g$-factors, and Luttinger parameters that are in good agreement with experimental data [44, 46, 48]. More important for the problems we consider, the band structures and linear optical absorption spectra are also in good agreement with experimental data. In Fig. 2 we show the relevant electronic bands for two different stoichiometries, and in Fig. 3 we show the imaginary parts of the corresponding dielectric functions, which are related to the 1-photon absorption rates (or carrier injection) by $\operatorname{Im} \varepsilon(\Omega)=\hbar \xi^{x x}(\Omega) / 2 \epsilon_{0}$. Calculations for $1+2$ QuIC in GaAs and Si have been computed [39] using the same $\boldsymbol{k} \cdot \boldsymbol{p}$ model and Density Functional Theory for comparison. For GaAs there is good agreement between the two models even for the non-diagonal spin injection tensors, and for Si the discrepancy between the two models is significant only at high frequencies. For GaAs the $\boldsymbol{k} \cdot \boldsymbol{p}$ model for the full BZ provides a good agreement with the Local Density Approximation, and for the frequencies we consider in $2+3 \mathrm{QuIC}$ we are restricted to the region of the $\mathrm{BZ}$ around the $\Gamma$ point, where even less sophisticated $\boldsymbol{k} \cdot \boldsymbol{p}$ models give good results.

As already mentioned, the 30-band model allows us to perform reliable calculations for total photon energies up to $6 \mathrm{eV}$. However, for the energies we are most interested - below $3 \mathrm{eV}$ - we can get accurate results for the optical absorption coefficients even if only 6 valence and 2 conduction bands are included in the model, and only the valley including the $\Gamma$ point of the $\mathrm{BZ}$ are considered. We also point out that the model we use is applicable to most zincblende semiconductors, where each specific material corresponds to a particular set of parameters. Since the bandstructures of zincblende semiconductors are qualitatively similar, our results presented in the next sections are qualitatively valid for most directgap zincblende semiconductors. 


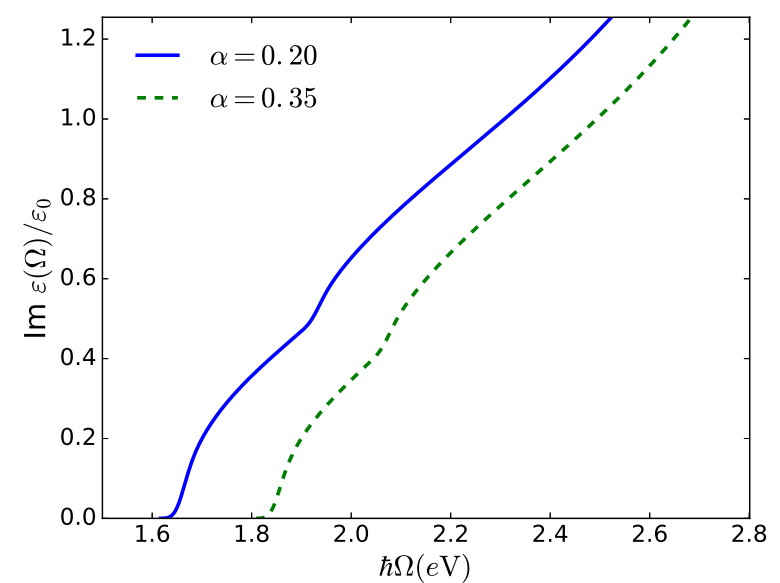

FIG. 3. Imaginary part of the dielectric function for two stoichiometries $\alpha$.

\section{QUANTUM INTERFERENCE CONTROL USING TWO- AND THREE-PHOTON ABSORPTION IN AlGaAs}

We consider two incident fields of different frequencies with amplitudes $\boldsymbol{E}_{\omega}=E_{\omega} e^{i \phi_{\omega}} \hat{\boldsymbol{e}}_{\omega}$ and $\boldsymbol{E}_{3 \omega / 2}=$ $E_{3 \omega / 2} e^{i \phi_{3 \omega / 2}} \hat{\boldsymbol{e}}_{3 \omega / 2}$, where $E_{\omega}>0$ and $E_{3 \omega / 2}>0$ are the field magnitudes, the unit vectors $\hat{\boldsymbol{e}}_{\omega}$ and $\hat{\boldsymbol{e}}_{3 \omega / 2}$ indicate their polarizations, and $\phi_{\omega}$ and $\phi_{3 \omega / 2}$ indicate their phases. We also define the phase parameter $\Delta \phi=$ $2 \phi_{3 \omega / 2}-3 \phi_{\omega}$, which will be useful later. We assume that the field at $3 \omega / 2$ has a weaker intensity than the field at $\omega$, and we demand that the frequencies satisfy $2 \omega<\Delta_{g}<3 \omega$, where $E_{g}=\hbar \Delta_{g}$ is the optical gap. Therefore only $3 \mathrm{PA}$ processes are important for the lower frequency field $\boldsymbol{E}_{\omega}$, while only $2 \mathrm{PA}$ processes are relevant for the higher frequency field $\boldsymbol{E}_{3 \omega / 2}$; the 3PA associated with $\boldsymbol{E}_{3 \omega / 2}$ is weaker due to the lower intensity of the field, and we neglect it.

We focus on $\mathrm{Al}$ concentrations $\alpha$ such that $0.18 \lesssim \alpha \lesssim$ 0.38 , since $\mathrm{Al}_{\alpha} \mathrm{Ga}_{1-\alpha}$ As with $\alpha$ too small has a band gap smaller than $2 \hbar \omega$ for telecommunication wavelengths ( $\hbar \omega \sim 0.8 \mathrm{eV}$ ), and $\mathrm{Al}_{\alpha} \mathrm{Ga}_{1-\alpha}$ As with $\alpha$ too large is too reactive.

\section{A. Carrier injection}

We track the number of injected carriers by calculating the number of electrons in the conduction bands, which corresponds to the operator

$$
\mathcal{N}=\sum_{c k} a_{c k}^{\dagger} a_{c k}
$$

so we use $n_{c c^{\prime}}=\delta_{c c^{\prime}}$ and $n_{v v^{\prime}}=0$ for the carrier density matrix elements in Eq. (19). The optical injection of carriers due to 2PA and 3PA processes, as well as their interference, is characterized by the tensors $\xi$, according
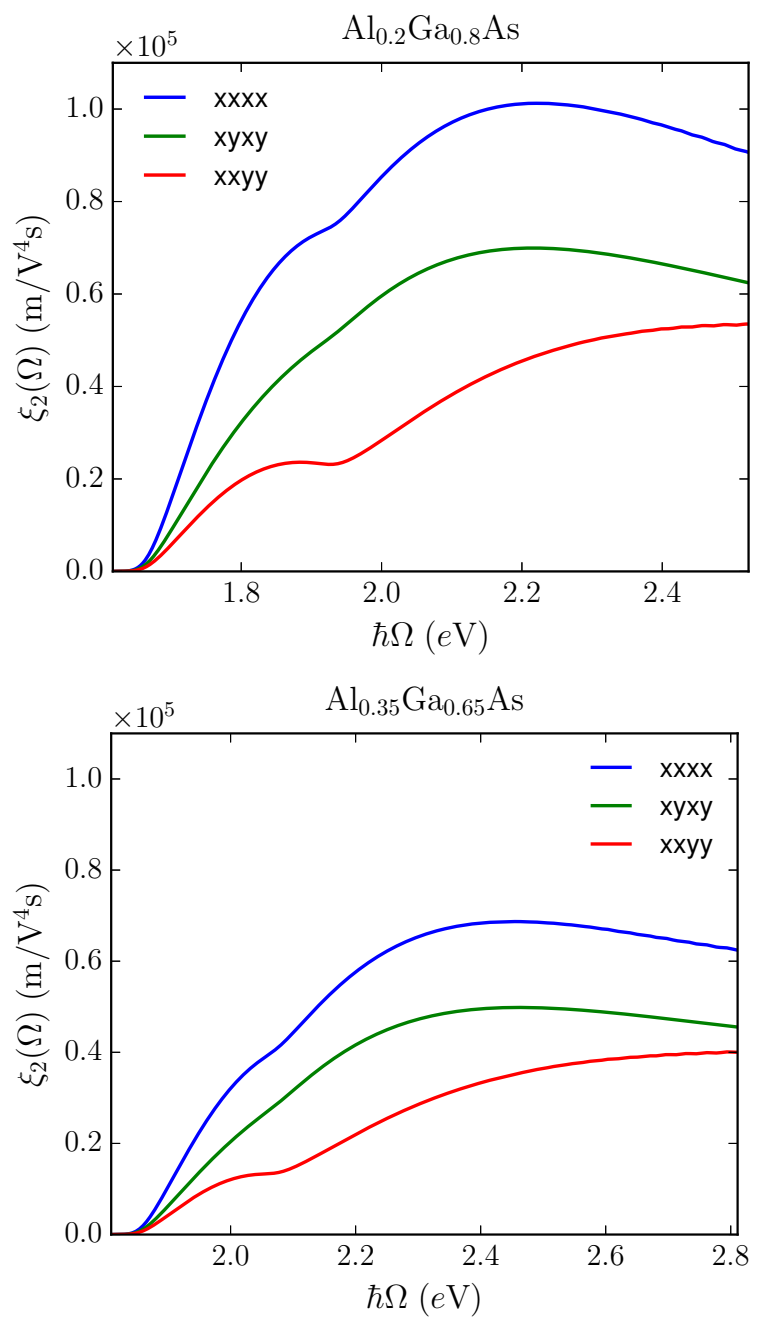

FIG. 4. Two-photon carrier injection coefficients for two stoichiometries.

to

$$
\begin{aligned}
\frac{d}{d t}\langle n\rangle_{2}= & \xi_{2}^{a b c d}(3 \omega) E_{-3 \omega / 2}^{a} E_{-3 \omega / 2}^{b} E_{3 \omega / 2}^{c} E_{3 \omega / 2}^{d}, \\
\frac{d}{d t}\langle n\rangle_{3}= & \xi_{3}^{a b c d e f}(3 \omega) E_{-\omega}^{a} E_{-\omega}^{b} E_{-\omega}^{c} E_{\omega}^{d} E_{\omega}^{e} E_{\omega}^{f}, \\
\frac{d}{d t}\langle n\rangle_{2+3(i)}= & \xi_{2+3}^{a b c d e}(3 \omega) E_{-\omega}^{a} E_{-\omega}^{b} E_{-\omega}^{c} E_{3 \omega / 2}^{d} E_{3 \omega / 2}^{e} \\
& + \text { c.c. }
\end{aligned}
$$




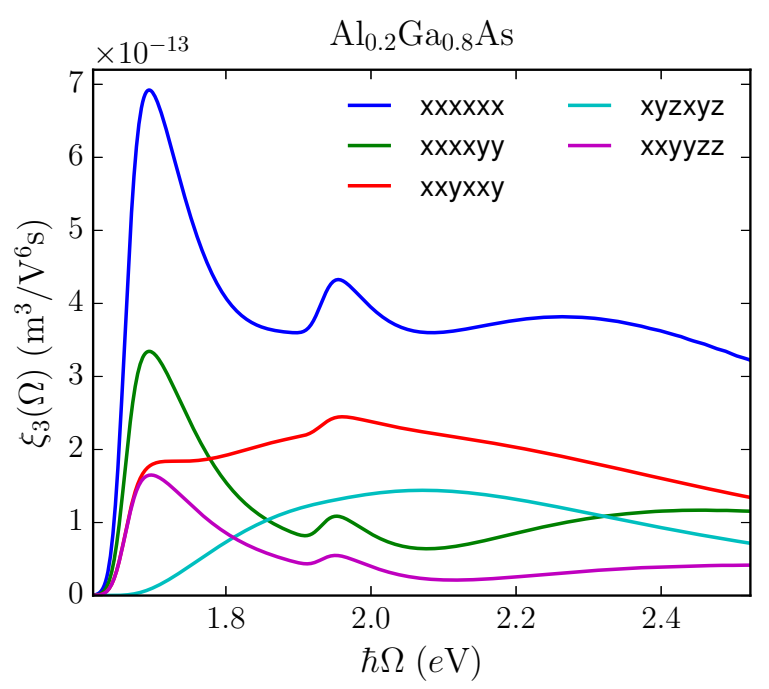

list the independent components of the injection tensor coefficients in Appendix A. In Figs. 4, 6 and 5, we show the frequency dependence of the independent components of the coefficients $\xi_{2}^{a b c d}(3 \omega), \xi_{3}^{a b c d e f}(3 \omega)$, and $\xi_{2+3}^{a b c d e}(3 \omega)$ respectively. Notice that the 3PA coefficient is large for frequencies right above the band gap, while the coefficient for 2PA nearly vanishes for similar frequencies. As discussed below Eqs. (17) and (18), the dominant contribution to $2 \mathrm{PA}$ always involves intraband velocity matrix elements, which correspond to the band dispersion, so they vanish at the $\Gamma$ point of the Brillouin zone. The 3PA has contributions from interband velocity matrix elements, which in general do not vanish at $\Gamma$.

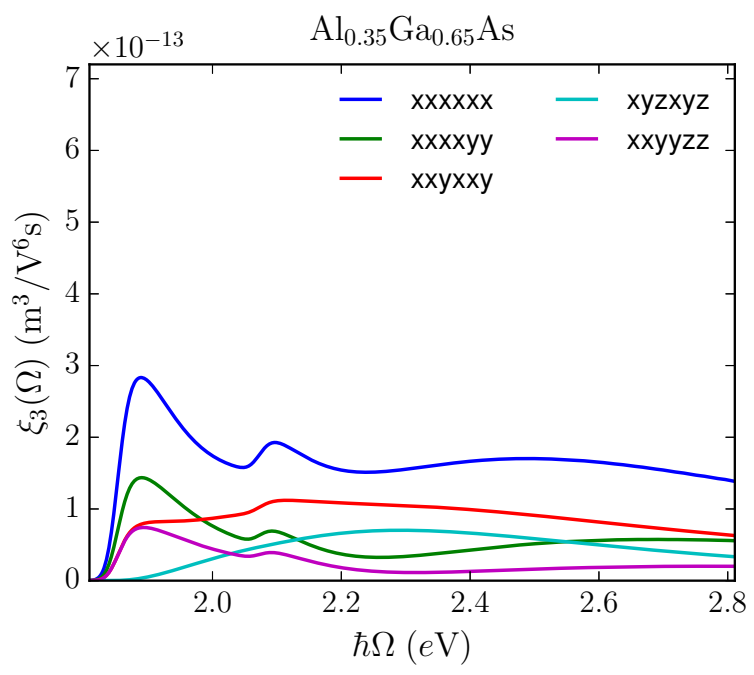

FIG. 5. Three-photon carrier injection coefficients for two stoichiometries.

where $\hbar \Omega=3 \hbar \omega$ is the total transition energy [49], and the coefficients are calculated as

$$
\begin{gathered}
\xi_{2}^{a b d e}(3 \omega)=2 \pi \int \frac{d \boldsymbol{k}}{(2 \pi)^{D}} \sum_{c v} R_{c v \boldsymbol{k}}^{a b *} R_{c v \boldsymbol{k}}^{d e} \delta\left(3 \omega-\omega_{c v}\right), \\
\xi_{3}^{a b d e f g}(3 \omega)=2 \pi \int \frac{d \boldsymbol{k}}{(2 \pi)^{D}} \sum_{c v} R_{c v \boldsymbol{k}}^{a b d *} R_{c v \boldsymbol{k}}^{e f g} \delta\left(3 \omega-\omega_{c v}\right), \\
\xi_{2+3}^{a b d e f}(3 \omega)=2 \pi \int \frac{d \boldsymbol{k}}{(2 \pi)^{D}} \sum_{c v} R_{c v \boldsymbol{k}}^{a b d *} R_{c v \boldsymbol{k}}^{e f} \delta\left(3 \omega-\omega_{c v}\right) .
\end{gathered}
$$

The $\xi_{2+3}^{a b d e f}(3 \omega)$ coefficient is associated with absorption processes described by $\chi^{(5)}$. The symmetries of the zincblende lattice, corresponding to the point group $T_{d}$ (or $43 \mathrm{~m}$ ), strongly restrict the number of independent non-zero components of the tensors $\xi_{2}, \xi_{2+3}$, and $\xi_{3}$. We
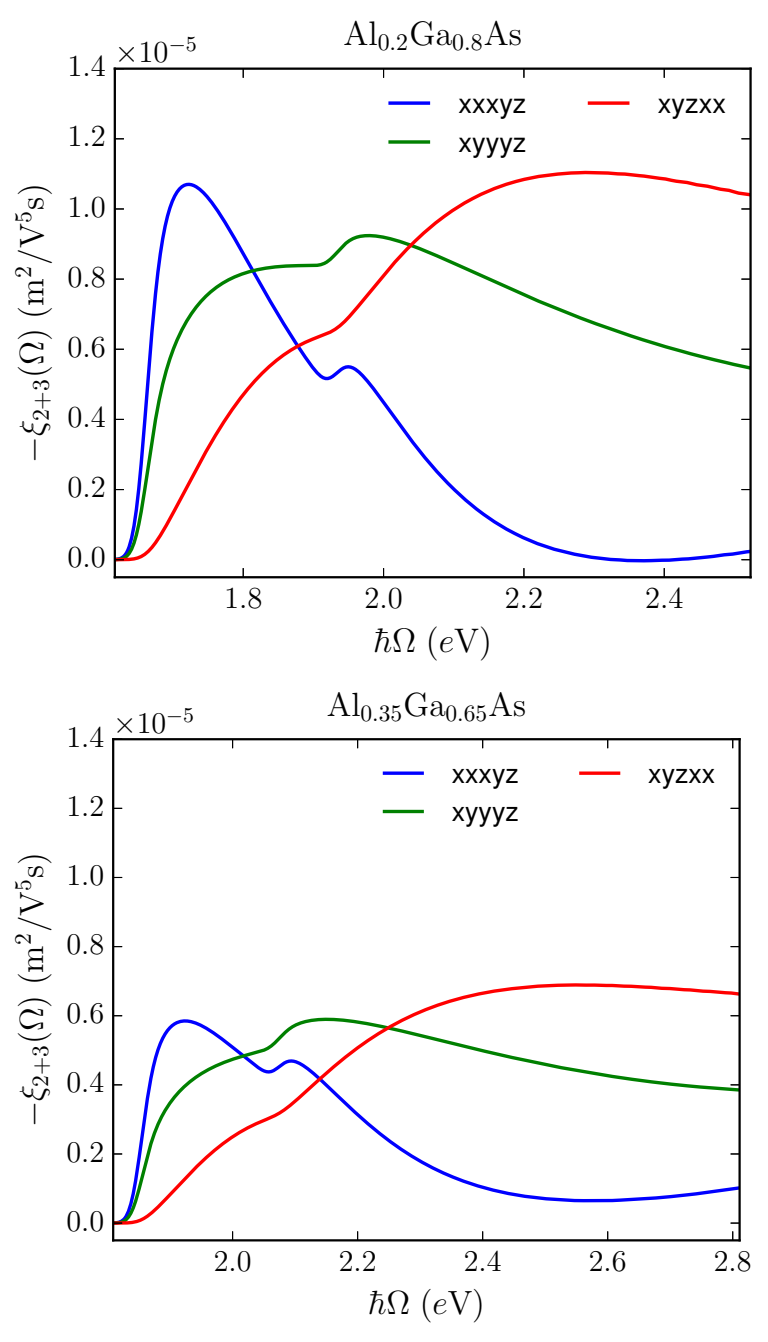

FIG. 6. Injection rate coefficients for carrier density corresponding to $2+3 \mathrm{QuIC}$ for two stoichiometries.

\section{B. Current injection}

Taking the quantity $\langle M\rangle$ in Eq. (15) to be the current density $\langle\boldsymbol{J}\rangle$, we can compute its injection rate due to the quantum interference between two- and three-photon 

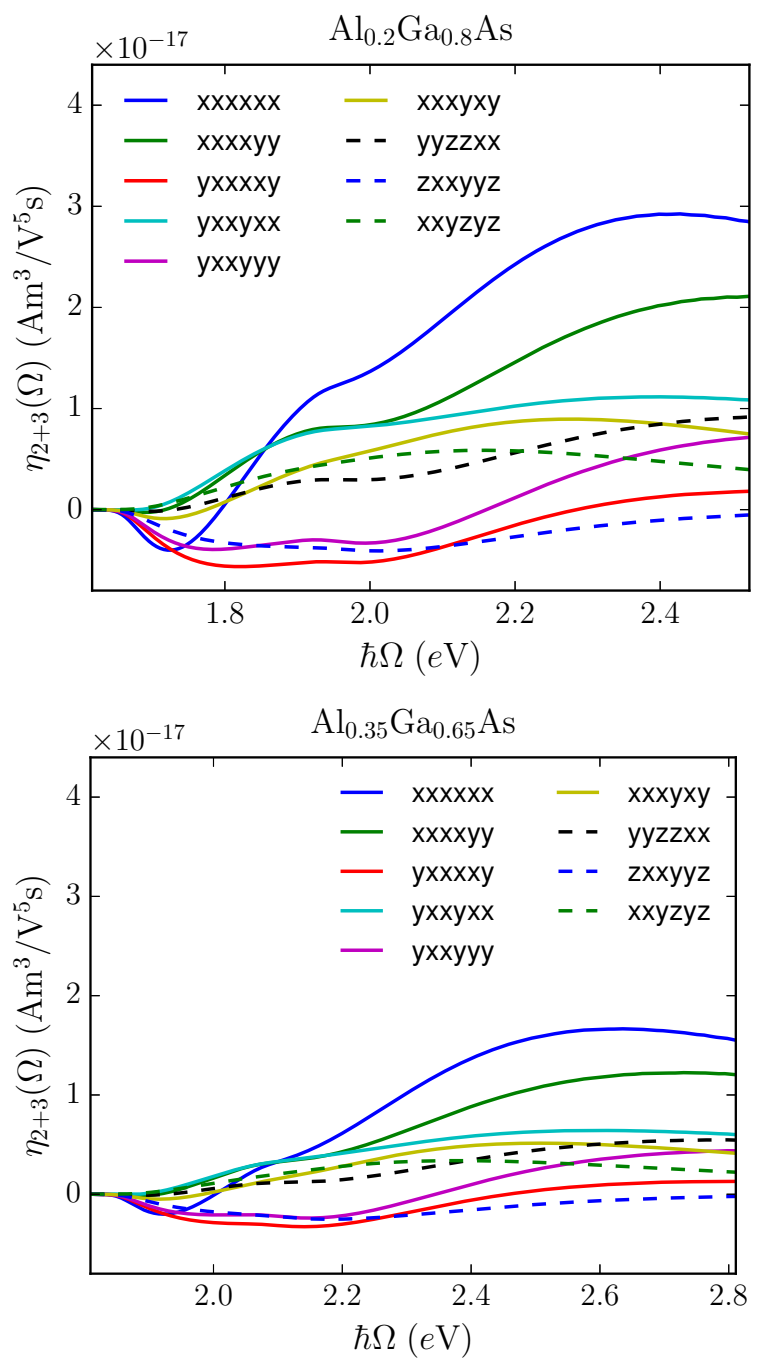

FIG. 7. Injection rate coefficients for current corresponding to $2+3$ QuIC for two stoichiometries.

absorption processes as

$\frac{d}{d t}\left\langle J^{a}\right\rangle_{2+3}=\eta_{2+3}^{a b c d e f}(3 \omega) E_{-\omega}^{b} E_{-\omega}^{c} E_{-\omega}^{d} E_{3 \omega / 2}^{e} E_{3 \omega / 2}^{f}+c . c .$,

where $\hbar \Omega=3 \hbar \omega$ is the total photon energy. We emphasize that $\eta_{2+3}^{\text {abcdef }}(3 \omega)$ is related to $\chi^{(5)}$ and is finite even for centro-symmetric materials. The dependence of the injected current on the fields is described by Eq. (30), which is the main equation to describe $2+3$ QuIC experiments being reported in the accompanying article [42]. In terms of intensities, Eq. (30) indicates that the current injection rate is proportional to the intensity of the $3 \omega / 2$ field, $I_{3 \omega / 2}$, and the intensity of the $\omega$ field to the power $3 / 2, I_{\omega}^{3 / 2}$. These dependences are verified in Fig. 4 of the accompanying experimental article [42], indicating that the currents measured in those experiments indeed correspond to $2+3 \mathrm{QuIC}$. We list the independent components of the injection tensor coefficient $\eta_{2+3}^{a b c d e f}(3 \omega)$ in
Appendix A. In Fig. 7 we show the frequency dependence of the independent components of the coefficient $\eta_{2+3}^{a b c d e f}(3 \omega)$ for different stoichiometries. The plots show that some components change sign as the frequency increases. This sign flip is due to the competing contributions due to intraband and interband velocity matrix elements to the $R_{c v k}^{(3)}$ coefficients. For low excess photon energies, the excited carriers are close to the $\Gamma$ point in the BZ, and the interband contribution is the most important, as the band dispersion is small. For larger photon excess energies, the excited carriers are located further from the $\Gamma$ point in the BZ, so the band dispersion is large and the intraband contributions are more important.
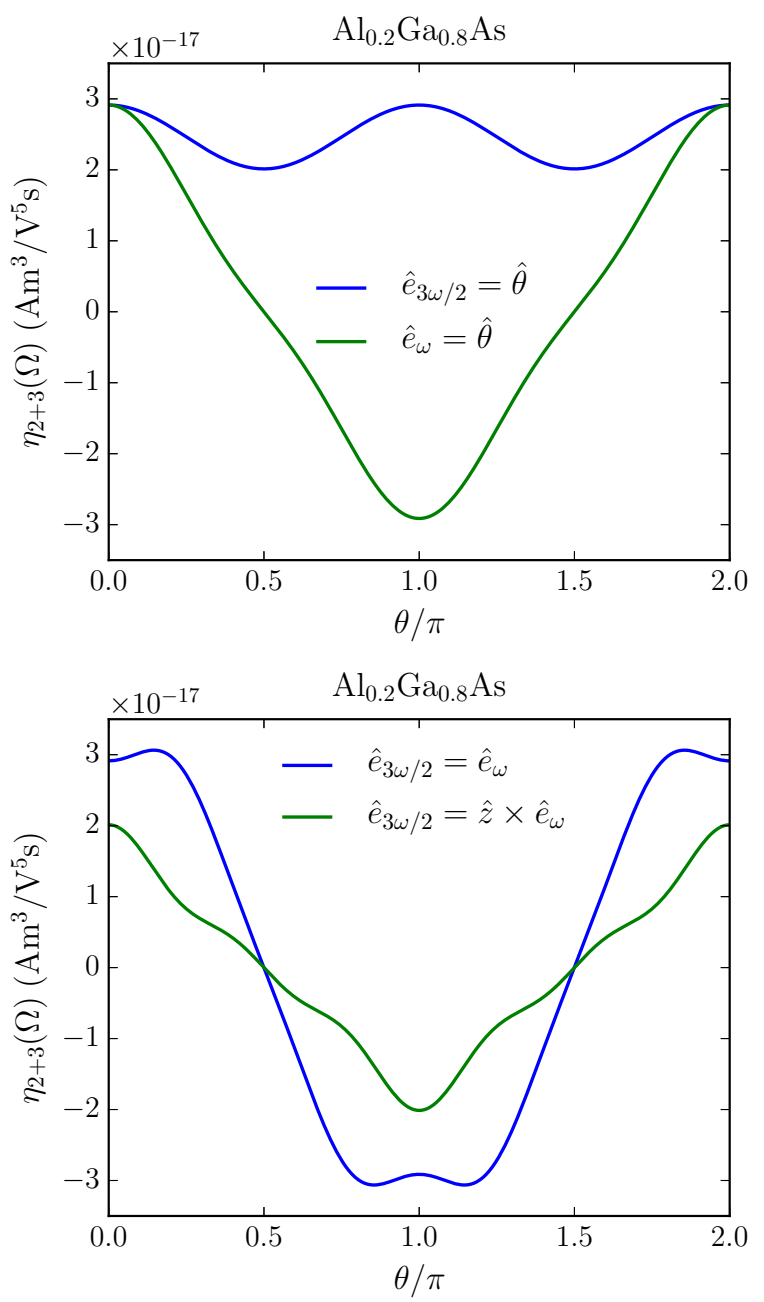

FIG. 8. Injection rate coefficient for current along the $\hat{\boldsymbol{x}}$ direction as the polarizations of the incident fields are rotated in the $\hat{\boldsymbol{x}}-\hat{\boldsymbol{y}}$ plane. (Top) Either $\hat{\boldsymbol{e}}_{\omega}$ or $\hat{\boldsymbol{e}}_{3 \omega / 2}$ is rotated while the other is fixed along the $\hat{\boldsymbol{x}}$ direction. (Bottom) In both cases $\hat{\boldsymbol{e}}_{\omega}=\hat{\boldsymbol{\theta}}$ is rotated, and $\hat{\boldsymbol{e}}_{3 \omega / 2}$ is either parallel or perpendicular to it. The total photon energy is $\hbar \Omega=2.4 \mathrm{eV}(\lambda \sim 520 \mathrm{~nm})$ in both cases.

To illustrate some aspects of the different tensor components, in Fig. 8 we plot the injection current for differ- 
ent polarizations of the incident fields in a typical experimental scenario. We assume that the sample has electrodes mounted such that they always measure the current along the [100] crystal direction, which we denote by $\hat{\boldsymbol{x}}$. In the first case we keep either $\hat{\boldsymbol{e}}_{\omega}$ or $\hat{\boldsymbol{e}}_{3 \omega / 2}$ fixed along the $\hat{\boldsymbol{x}}$ direction, while the other field is rotated in the $\hat{\boldsymbol{x}}-\hat{\boldsymbol{y}}$ plane and points along the direction $\hat{\boldsymbol{\theta}}=\hat{\boldsymbol{x}} \cos \theta+\hat{\boldsymbol{y}} \sin \theta$, where $\hat{\boldsymbol{y}}$ corresponds to the [010] crystal direction. The case where $\hat{\boldsymbol{e}}_{\omega}=\hat{\boldsymbol{\theta}}$ and $\hat{\boldsymbol{e}}_{3 \omega / 2}=\hat{\boldsymbol{x}}$, corresponding to the green line in Fig. 8, is tested experimentally and reported in Fig. 5 of the accompanying paper [42]. In the second scenario, the polarizations of both incident fields are rotated in the $\hat{\boldsymbol{x}}-\hat{\boldsymbol{y}}$ plane and they are kept either parallel or perpendicular to each other. In Fig. 8, we show that the current is largely along the $\hat{\boldsymbol{e}}_{\omega}$ direction regardless of the $\hat{\boldsymbol{e}}_{3 \omega / 2}$ direction. However, the magnitude of the current depends significantly on the $\hat{\boldsymbol{e}}_{3 \omega / 2}$ direction, and it is maximal for $\hat{\boldsymbol{e}}_{3 \omega / 2}=\hat{\boldsymbol{e}}_{\omega}$.

\section{Swarm velocity}

Since the excited carriers respond to the induced voltage due to the injected current, and usually screen it at least partially, a good measure of the efficiency of the current injection is the swarm velocity, defined as $\boldsymbol{v}_{\text {swarm }}=\frac{d}{d t}\langle\boldsymbol{J}\rangle / e \frac{d}{d t}\langle n\rangle$, which represents the average contribution to the injection current due to one excited electron [50]. Since $\langle n\rangle_{2+3} \ll\langle n\rangle_{2}+\langle n\rangle_{3}$, the total density of carriers is $\langle n\rangle \simeq\langle n\rangle_{2}+\langle n\rangle_{3}$, and for both light beams polarized along the $\hat{\boldsymbol{x}}$ direction we have a swarm velocity of magnitude

$$
v_{\mathrm{swarm}}=\frac{2\left|\eta_{2+3}^{x x x x x}(3 \omega)\right| E_{\omega}^{3} E_{3 \omega / 2}^{2}}{|e|\left(\xi_{3}^{x x x x x x}(3 \omega) E_{\omega}^{6}+\xi_{2}^{x x x x}(3 \omega) E_{3 \omega / 2}^{4}\right)},
$$

where we have chosen $\Delta \phi=\pi / 2$ to optimize the magnitude of the numerator. The whole expression is optimized by choosing the intensities of the two beams appropriately; the condition to be satisfied is $\xi_{2}^{x x x x}(3 \omega) E_{3 \omega / 2}^{4}=$ $\xi_{3}^{x x x x x x}(3 \omega) E_{\omega}^{6}$, which corresponds to an equal number of carriers injected by 2-photon absorption and 3-photon absorption. If this holds,

$$
v_{\mathrm{swarm}}=\frac{\left|\eta_{2+3}^{x x x x x x}(3 \omega)\right|}{|e| \sqrt{\xi_{3}^{x x x x x x}(3 \omega) \xi_{2}^{x x x x}(3 \omega)}} .
$$

In Fig. 9 we plot this expression, together with the expression that would result if the beam of frequency $\omega$ were polarized in the $\hat{\boldsymbol{x}}$ direction while the one of frequency $3 \omega / 2$ in the $\hat{\boldsymbol{y}}$ direction, which is the same as Eq. (32) but with $\eta_{2+3}^{x x x x x x}(3 \omega)$ replaced by $\eta_{2+3}^{x x x y y}(3 \omega)$; as well, $\xi_{2}^{x x x x}(3 \omega)$ should also be replaced by $\xi_{2}^{y y y y}(3 \omega)$, but they are equal. We see that different stoichiometries give similar values for the swarm velocity if the frequency is adjusted according to the band gap of the system. The fact that higher Ga concentrations lead to larger injected
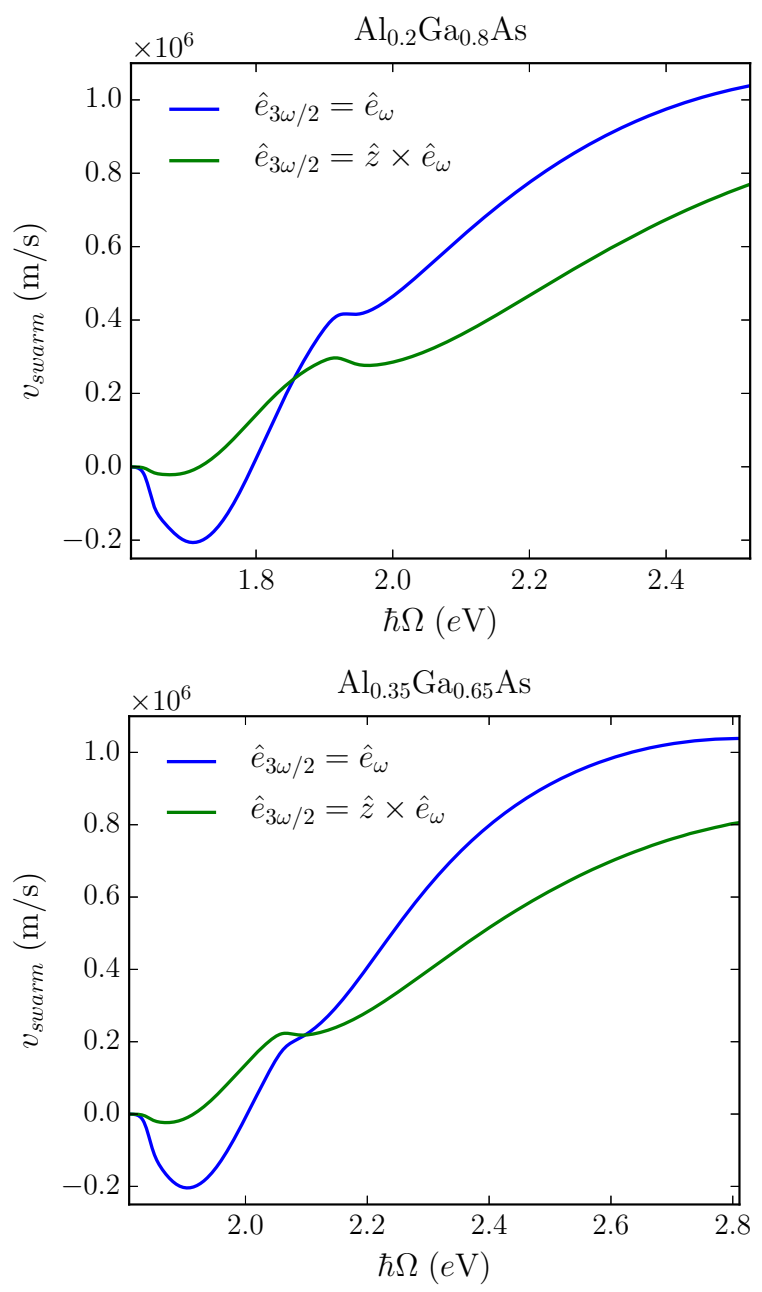

FIG. 9. Swarm velocity for two stoichiometries assuming optimum interference between $2 \mathrm{PA}$ and $3 \mathrm{PA}$. The current is measured along the $\hat{\boldsymbol{x}}$ direction, and so is the polarization of the lower frequency field $\hat{\boldsymbol{e}}_{\omega}=\hat{\boldsymbol{x}}$, while we consider two cases for the polarization of the higher frequency field: $\hat{\boldsymbol{e}}_{3 \omega / 2}=\hat{\boldsymbol{x}}$ and $\hat{\boldsymbol{e}}_{3 \omega / 2}=\hat{\boldsymbol{y}}$.

currents (see Fig. 7) is only due to a higher carrier injection. Yet with appropriate laser intensities it is possible to reach the same levels of injected current densities with any $\mathrm{Al}$ concentration, although the laser frequencies and intensities at which the maximum is achieved depend on the $\mathrm{Al}$ concentration.

We point out that the $2+3$ QuIC swarm velocity is about twice its equivalent for $1+2 \mathrm{QuIC}$. This is an indication that the distribution of carriers injected in the BZ is sharper for $2+3$ QuIC compared to $1+2$ QuIC. We further confirm that by computing the variance of the lattice momentum $\boldsymbol{k}$ of the electrons injected in the conduction band for both 1+2 QuIC, $\sigma_{1+2}^{a}=\left\langle\left(k^{a}\right)^{2}\right\rangle_{1+2}-\left\langle k^{a}\right\rangle_{1+2}^{2}$, and $2+3$ QuIC, $\sigma_{2+3}^{a}=\left\langle\left(k^{a}\right)^{2}\right\rangle_{2+3}-\left\langle k^{a}\right\rangle_{2+3}^{2}$. For the incident fields polarized along the $\hat{\boldsymbol{x}}$ direction, we 
find $\langle\boldsymbol{k}\rangle_{1+2}=(4.9,0,0) \times 10^{-2} \AA^{-1}$ and $\langle\boldsymbol{k}\rangle_{2+3}=$ $(5.7,0,0) \times 10^{-2} \AA^{-1}$, as well as $\sigma_{1+2}=(3.4,4.3,4.3) \times$ $10^{-3} \AA^{-2}$ and $\sigma_{2+3}=(2.8,2.2,2.2) \times 10^{-3} \AA^{-2}$, which indeed indicates that the distribution of injected electrons in the BZ is sharper for $2+3$ QuIC, especially in the directions transverse $(\hat{\boldsymbol{y}}$ and $\hat{\boldsymbol{z}})$ to the polarization of the field.

\section{Laser intensities}

Our calculations are performed in the perturbative regime, the validity of which requires that the fraction of the injected carrier population density relative to the total density of states $n_{\max }$ in the range of energies covered by the laser pulse be small. We thus consider our calculations to be valid when

$$
\langle n\rangle_{2}+\langle n\rangle_{3}<0.1 n_{\max },
$$

where the fraction 0.1 is chosen somewhat arbitrarily. The carrier injection due to the 2- and 3-photon interference $\langle n\rangle_{2+3(i)}$ mostly has the effect of concentrating the carrier injection in some region of the $\mathrm{BZ}$, but it does not contribute significantly to the total number of injected carriers compared to $\langle n\rangle_{2}$ and $\langle n\rangle_{3}$. For the estimates of laser intensities we consider the incident fields to be both polarized along the $\hat{\boldsymbol{x}}$ direction, so for a laser pulse of duration $\mathcal{T}$ we require

$$
\begin{array}{r}
{\left[\frac{d}{d t}\langle n\rangle_{2}+\frac{d}{d t}\langle n\rangle_{3}\right] \mathcal{T}<0.1 n_{\max },} \\
{\left[\xi_{2}^{x x x x}(3 \omega) E_{3 \omega / 2}^{4}+\xi_{3}^{x x x x x x}(3 \omega) E_{\omega}^{6}\right] \mathcal{T}<0.1 n_{\max } .}
\end{array}
$$

The maximum density of states $n_{\max }$ that can be injected is determined by analyzing the volume $\mathrm{V}$ corresponding to the excited states in the BZ. We denote by $k_{\Omega}$ the momentum corresponding to the energy difference $\hbar \omega_{c v \boldsymbol{k}}=\hbar \Omega$ between the conduction and valence bands, so $\mathrm{V}=4 \pi k_{\Omega}^{2} \Delta k$, where $\Delta k=\frac{d k}{d \omega_{c v k}} \Delta \omega_{c v \boldsymbol{k}}$ is related to the frequency broadening $\Delta \omega=2 \pi / \mathcal{T}$ associated with the time duration of the pulse. The derivative of the band energy corresponds to the velocities of electrons in the conduction and valence bands, $v_{\Omega}=\frac{d \omega_{c v}}{d k}=\frac{d \omega_{c}}{d k}-\frac{d \omega_{v}}{d k}$, so $\mathrm{V}=8 \pi^{2} k_{\Omega}^{2} / v_{\Omega} \mathcal{T}$. The volume in the $\mathrm{BZ}$ associated with one quantum state is $\mathrm{V}_{1}=(2 \pi / L)^{3}$, where $L$ is the normalization length of the sample. The number of states that can be excited is then $\mathrm{V} / \mathrm{V}_{1}$, and their spatial density is

$$
n_{\max }=\frac{\mathrm{V}}{\mathrm{V}_{1} L^{3}}=\frac{k_{\Omega}^{2}}{\pi v_{\Omega} \mathcal{T}} .
$$

For optimal interference, there should be equal densities of carriers injected by 2 - and 3-photon absorption, $\langle n\rangle_{2}=$ $\langle n\rangle_{3}$, which according to Eq. (35) gives

$$
\xi_{2}^{x x x x}(3 \omega) E_{3 \omega / 2}^{4}=\xi_{3}^{x x x x x x}(3 \omega) E_{\omega}^{6}<0.05 \frac{k_{\Omega}^{2}}{\pi v_{\Omega} \mathcal{T}^{2}} .
$$

The maximal amplitudes $E_{\omega}$ and $E_{3 \omega / 2}$ of the incident fields can then be estimated from the extreme of the inequality in the above equation. For the stoichiometry of $\alpha=0.2$, pulses with duration $\mathcal{T}=150 \mathrm{fs}$, and total photon energy $\hbar \Omega=2.4 \mathrm{eV}$, we have

$$
\begin{aligned}
E_{\omega} & =1.24 \times 10^{8} \frac{\mathrm{V}}{\mathrm{m}}, \\
E_{3 \omega / 2} & =6.05 \times 10^{7} \frac{\mathrm{V}}{\mathrm{m}} .
\end{aligned}
$$

The intensities in the material medium with these field amplitudes are

$$
\begin{aligned}
I_{\omega} & =2 \epsilon_{0} c n_{\omega} E_{\omega}^{2}=26.5 \frac{\mathrm{GW}}{\mathrm{cm}^{2}}, \\
I_{3 \omega / 2} & =2 \epsilon_{0} c n_{3 \omega / 2} E_{3 \omega / 2}^{2}=6.54 \frac{\mathrm{GW}}{\mathrm{cm}^{2}} .
\end{aligned}
$$

For these values, the injected current density is

$$
\left\langle J^{x}\right\rangle=2 \eta_{2+3}^{x x x x x x}(3 \omega) E_{\omega}^{3} E_{3 \omega / 2}^{2} \mathcal{T}=6.25 \frac{\mathrm{MA}}{\mathrm{cm}^{2}} .
$$

We emphasize that these are just estimates, as the limit of carrier density is set somewhat arbitrarily in Eq. (33). We note that we are ignoring scattering of the injected carriers. This means that the true maximal intensities would be larger than our estimates here, since there is room for more photon absorption as scattering depletes some of the excited states. We also note that in this treatment the electron-electron interaction has been neglected; were it included, the phase parameter would be shifted. However this shift is usually very small for zincblende semiconductors, except for frequencies very close to the band gap [51].

\section{DISCUSSION AND CONCLUSION}

One of the main utilities of QuIC in semiconductors is the injection of carriers in localized regions of the BZ. In this respect $2+3 \mathrm{QuIC}$ performs better than $1+2$ $\mathrm{QuIC}$, and that can be seen in a higher swarm velocity of $2+3 \mathrm{QuIC}$, which is a desirable feature for current injection. Another interesting difference between $1+2$ and $2+3 \mathrm{QuIC}$ is that in $2+3$ QuIC several current injection coefficients change sign as the total photon energy is increased, while in $1+2$ QuIC they typically do not. This happens because interband velocity matrix elements are responsible for the largest contribution to the $3 \mathrm{PA}$ coefficient at low photon energies, but at higher photon energies the intraband velocity matrix elements dominate. Since only nonlinear optical processes are involved in $2+3 \mathrm{QuIC}$, the laser intensities required for maximal 
effect are higher than for 1+2 QuIC, but still moderate. Also, the optical fields have a power law attenuation as they propagate through the absorbing material, instead of the exponential attenuation of linear absorption. Thus a waveguide geometry is desirable, and while QuIC in waveguides presents some challenges, as it raises issues of phase- and mode-matching, it also presents opportunities for easy integration with devices on-chip. Since optical frequency combs are routinely propagated through waveguides, there should be no additional difficulties for $2+3$ QuIC experiments in waveguides other than the usual issues of phase- and mode-matching.

\section{ACKNOWLEDGMENT}

We thank Perry T. Mahon for useful discussions. This work has been supported by DARPA through the DODOS program.

\section{Appendix A: Nonzero injection coefficient components of zincblende lattices}

AlGaAs in the virtual crystal approximation forms a zincblende lattice, which has the symmetry of point group $T_{d}$ (or $43 \mathrm{~m}$ ). The optical responses we consider in this work involve tensors of rank 4 up to 6 . With $T_{d}$ symmetries [52], generic rank-4 tensors have 21 nonzero components of which 4 are independent, rank- 5 tensors have 60 non-zero and 10 independent components, and rank- 6 tensors have 183 non-zero and 31 independent components. However, the tensors representing the optical processes have a few more specific restrictions due to their relation to the optical fields, as the indices associated with the same incident field are symmetrized.

With these considerations the tensor $\xi_{2}$ has 3 independent components

$$
\begin{aligned}
& \xi_{2}^{x x x x}=P(x, y, z), \\
& \xi_{2}^{x y x y}=\xi_{2}^{x y y x}=P(x, y, z), \\
& \xi_{2}^{x x y y}=P(x, y, z),
\end{aligned}
$$

where $P(x, y, z)$ indicates all the possible permutations of $(x, y, z)$ in the indices. The tensor $\xi_{2+3}$ has 3 independent components

$$
\begin{aligned}
\xi_{2+3}^{x x x y z} & =P(x, y, z), \\
\xi_{2+3}^{x x y x z} & =\xi_{2+3}^{x x y z x}=\xi_{2+3}^{x y x x z} \\
& =\xi_{2+3}^{x y x z x}=\xi_{2+3}^{y x x x z}=\xi_{2+3}^{y x x z x}=P(x, y, z), \\
\xi_{2+3}^{x y z x x} & =\xi_{2+3}^{y x z x x}=\xi_{2+3}^{y z x x x}=P(x, y, z),
\end{aligned}
$$

and $\xi_{3}$ has 5 independent components

$$
\begin{aligned}
\xi_{3}^{x x x x x x} & =P(x, y, z), \\
\xi_{3}^{x x x x y y} & =\xi_{3}^{x x x y x y}=\xi_{3}^{x x x y y x} \\
& =\xi_{3}^{y y x x x x}=\xi_{3}^{y x y x x x}=\xi_{3}^{x y y x x x}=P(x, y, z), \\
\xi_{3}^{x x y x x y} & =\xi_{3}^{x x y x y x}=\xi_{3}^{x x y y x x}=\xi_{3}^{x y x x x y}=\xi_{3}^{x y x x y x}=\xi_{3}^{x y x y x x} \\
& =\xi_{3}^{y x x x x y}=\xi_{3}^{y x x x y x}=\xi_{3}^{y x x y x x}=P(x, y, z), \\
\xi_{3}^{x x y y z z} & =\xi_{3}^{x x y z y z}=\xi_{3}^{x x y z z y}=\xi_{3}^{x y x y z z}=\xi_{3}^{x y x y z y z}=\xi_{3}^{x y x z z y} \\
& =\xi_{3}^{y x x y z z}=\xi_{3}^{y x x y z y z}=\xi_{3}^{y x x z z y}=P(x, y, z), \\
\xi_{3}^{x y z x y z} & =\xi_{3}^{x y z z x y}=\xi_{3}^{x y z y z x}=\xi_{3}^{x y z z y x} \\
& =\xi_{3}^{x y z x z y}=\xi_{3}^{x y z y x z}=P(\mathrm{~A} 10)
\end{aligned}
$$

Finally, the tensor $\eta_{2+3}$ has 9 independent components

$$
\begin{aligned}
\eta_{2+3}^{x x x x x} & =P(x, y, z), \\
\eta_{2+3}^{x x x y y} & =P(x, y, z), \\
\eta_{2+3}^{x x y y x x} & =\eta_{2+3}^{x y x y x x}=\eta_{2+3}^{x y y x x x}=P(x, y, z), \\
\eta_{2+3}^{x x x y x y} & =\eta_{2+3}^{x x y y x}=\eta_{2+3}^{x x y x x y} \\
& =\eta_{2+3}^{x x y x y x}=\eta_{2+3}^{x y x x x y}=\eta_{2+3}^{x y x x y x}=P(x, y, z), \\
\eta_{2+3}^{y x x x x y} & =\eta_{2+3}^{y x x x y x}=P(x, y, z), \\
\eta_{2+3}^{y x x y x x} & =\eta_{2+3}^{y x y x x x}=\eta_{2+3}^{y y x x x x}=P(x, y, z), \\
\eta_{2+3}^{x x y y z z} & =\eta_{2+3}^{x y x y z z}=\eta_{2+3}^{x y y x z z}=P(x, y, z), \\
\eta_{2+3}^{x x y z y z} & =\eta_{2+3}^{x x z y y z}=\eta_{2+3}^{x y x z y z} \\
& =\eta_{2+3}^{x y z x y z}=\eta_{2+3}^{x z x y y z}=\eta_{2+3}^{x z y x y z}=P(x, y, z) \\
& =\eta_{2+3}^{x y y z z x}=\eta_{2+3}^{x y z y z x}=\eta_{2+3}^{x z y y z x}=P(x, y, z) \\
\eta_{2+3}^{x y y z x z} & =\eta_{2+3}^{x y z y x z}=\eta_{2+3}^{x z y y x z} \\
&
\end{aligned}
$$

The independent components shown here are the ones plotted in the figures in the main text.
[1] E. A. Manykin, Laser Physics 11, 60 (2001).

[2] M. Shapiro and P. Brumer, Principles of the Quantum
Control of Molecular Processes (Wiley-Interscience, New York, 2003). 
[3] P. Brumer and M. Shapiro, Chemical Physics Letters 126, 541 (1986).

[4] C. Chen, Y.-Y. Yin, and D. S. Elliott, Physical Review Letters 64, 507 (1990).

[5] L. Zhu, V. Kleiman, X. Li, S. P. Lu, K. Trentelman, and R. J. Gordon, Science 270, 77 (1995).

[6] H. Nagai, H. Ohmura, F. Ito, T. Nakanaga, and M. Tachiya, J. Chem. Phys. 124, 034304 (2006).

[7] G. Kurizki, M. Shapiro, and P. Brumer, Physical Review B 39, 3435 (1989).

[8] N. B. Baranova, A. N. Chudinov, and B. Y. Zel'dovich, Optics Communications 79, 116 (1990).

[9] N. M. Lawandy, Optics Communications 85, 369 (1991).

[10] N. B. Baranova, A. N. Chudinov, and B. Y. Zel'dovich, Optics Communications 85, 371 (1991).

[11] E. Dupont, P. B. Corkum, H. C. Liu, M. Buchanan, and Z. R. Wasilewski, Physical Review Letters 74, 3596 (1995).

[12] R. Atanasov, A. Hache, J. L. P. Hughes, H. M. van Driel, and J. E. Sipe, Phys. Rev. Lett. 76, 1703 (1996).

[13] A. Hache, Y. Kostoulas, R. Atanasov, J. J. P. Hughes, and J. E. Sipe, Phys. Rev. Lett. 78, 306 (1997).

[14] J. Rioux and J. E. Sipe, Physica E 45, 1 (2012).

[15] D. Sun, C. Divin, J. Rioux, J. E. Sipe, C. Berger, W. A. de Heer, P. N. First, and T. B. Norris, Nano Lett. 10, 1293 (2010).

[16] J. Rioux, G. Burkard, and J. E. Sipe, Phys. Rev. B 83, 195406 (2011).

[17] K. M. Rao and J. E. Sipe, Phys. Rev. B 86, 115427 (2012).

[18] R. A. Muniz and J. E. Sipe, Phys. Rev. B 89, 205113 (2014).

[19] D. A. Bas, K. Vargas-Velez, S. Babakiray, T. A. Johnson, P. Borisov, T. D. Stanescu, D. Lederman, and A. D. Bristow, Appl. Phys. Lett. 106, 041109 (2015).

[20] D. A. Bas, R. A. Muniz, S. Babakiray, D. Lederman, J. E. Sipe, and A. D. Bristow, Opt. Express 24, 23583 (2016).

[21] R. A. Muniz and J. E. Sipe, Phys. Rev. B 91, 085404 (2015).

[22] Q. Cui and H. Zhao, ACS Nano 9, 3935 (2015).

[23] P. T. Mahon, R. A. Muniz, and J. E. Sipe, arXiv:1810.09971 [cond-mat.mes-hall] (2018).

[24] R. D. R. Bhat and J. E. Sipe, Phys. Rev. Lett. 85, 5432 (2000).

[25] M. J. Stevens, A. L. Smirl, R. D. R. Bhat, J. E. Sipe, and H. M. van Driel, J. Appl. Phys. 91, 4382 (2002).

[26] M. J. Stevens, A. L. Smirl, R. D. R. Bhat, A. Najmaie, J. E. Sipe, and H. M. van Driel, Phys. Rev. Lett. 90, 136603 (2003).

[27] J. Hubner, W. W. Ruhle, M. Klude, D. Hommel, R. D. R. Bhat, J. E. Sipe, and H. M. van Driel, Phys. Rev. Lett. 90, 216601 (2003).

[28] H. Zhao, E. J. Loren, H. M. van Driel, and A. L. Smirl, Phys. Rev. Lett. 96, 246601 (2006).

[29] C. Salazar, J. L. Cheng, and J. E. Sipe, Phys. Rev. B 93, 075442 (2016).

[30] K. Behnia, Nature Nanotechnology 7, 488 (2012).
[31] P. A. Roos, X. Li, R. P. Smith, J. A. Pipis, T. M. Fortier, and S. T. Cundiff, Opt. Lett. 30, 735 (2005).

[32] P. A. Roos and S. T. Cundiff, Laser Physics 15, 769 (2005).

[33] R. Smith, P. A. Roos, J. K. Wahlstrand, J. A. Pipis, M. B. Rivas, and S. T. Cundiff, J. Res. Nat. Inst. Sci. Tech. 112, 289 (2007).

[34] P. A. Roos, X. Li, J. R. Pipis, and S. T. Cundiff, Opt. Exp. 12, 4225 (2004).

[35] T. M. Fortier, P. A. Roos, D. J. Jones, S. T. Cundiff, R. D. R. Bhat, and J. E. Sipe, Phys. Rev. Lett. 92, 147403 (2004).

[36] S. T. Cundiff and J. Ye, Rev. Mod. Phys. 75, 325 (2003).

[37] J. Ye and S. Cundiff, Femtosecond Optical Frequency Comb: Principle, Operation, and Applications (Springer, Norwell, MA, 2005).

[38] P. A. Roos, X. Li, J. A. Pipis, T. M. Fortier, S. T. Cundiff, R. D. R. Bhat, and J. E. Sipe, J. Opt. Soc. Am. B 22, 362 (2005).

[39] F. Nastos, J. Rioux, M. Strimas-Mackey, B. S. Mendoza, and J. E. Sipe, Phys. Rev. B 76, 205113 (2007).

[40] E. Sternemann, T. Jostmeier, C. Ruppert, H. T. Duc, T. Meier, and M. Betz, Phys. Rev. B 88, 165204 (2013).

[41] E. Sternemann, T. Jostmeier, C. Ruppert, S. Thunich, H. T. Duc, R. Podzimski, T. Meier, and M. Betz, Applied Physics B 122, 1 (2016).

[42] K. Wang, R. A. Muniz, J. E. Sipe, and S. T. Cundiff, arXiv:1808.07523 [cond-mat.mes-hall] (2018).

[43] The denominators in Eqs. (17) and (18) do not lead to any divergences because of the assumption that $2 \hbar \omega$ is below the gap.

[44] S. Richard, F. Aniel, and G. Fishman, Phys. Rev. B 70, 235204 (2004).

[45] C. Salazar, R. A. Muniz, and J. E. Sipe, Phys. Rev. Materials 1, 054006 (2017).

[46] N. Fraj, I. Saidi, S. B. Radhia, and K. Boujdaria, J. Appl. Phys. 102, 053703 (2007).

[47] The corrections that are quadratic on the stoichiometry parameter $\alpha$ are small, and do not lead to significant changes in the band energies within a tolerance given by room temperature.

[48] J. Rioux, Full-band structure calculations of optical injection in semiconductors: Investigations of one-color, twocolor, and pump-probe scenarios, Ph.D. thesis, University of Toronto (2011).

[49] If the band gap of the material is smaller than $5 \omega / 2$ there would be an additional contribution to $n_{2}$ proportional to $E_{-\omega} E_{-3 \omega / 2} E_{\omega} E_{3 \omega / 2}$, but these carriers do not contribute to the interference between $2 \mathrm{PA}$ and $3 \mathrm{PA}$, which is our main interest.

[50] Notice that assuming constant injection rates, which is a reasonable assumption at least during some time interval, the definition of the swarm velocity is equivalent to $\left.\mathbf{v}_{\text {swarm }}=\langle\mathbf{J}\rangle / e<n\right\rangle$.

[51] R. D. R. Bhat and J. E. Sipe, Phys. Rev. B 72, 075205 (2005).

[52] E. G. Sauter, Nonlinear Optics (Wiley-Interscience, New York, 1996). 